
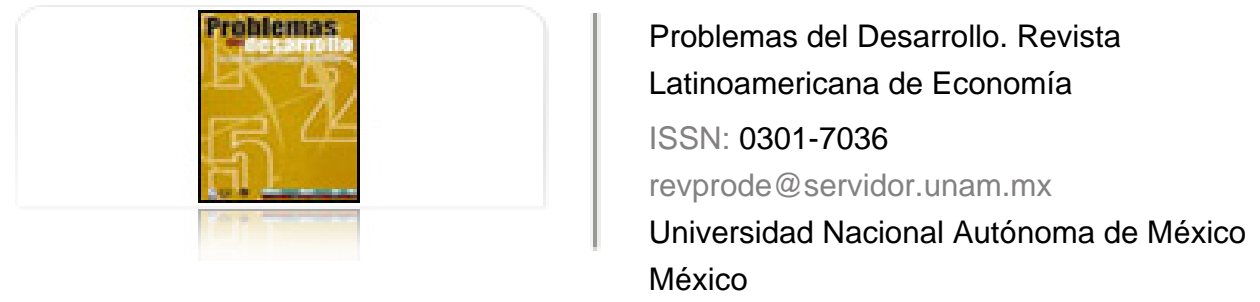

Rodríguez Benavides, Domingo; López Herrera, Francisco DESARROLLO FINANCIERO Y CRECIMIENTO ECONÓMICO EN MÉXICO Problemas del Desarrollo. Revista Latinoamericana de Economía, vol. 40, núm. 159, octubrediciembre, 2009, pp. 39-60

Universidad Nacional Autónoma de México

Distrito Federal, México

Disponible en: http://www.redalyc.org/articulo.oa?id=11820103003

- Cómo citar el artículo

- Número completo

- Más información del artículo

- Página de la revista en redalyc.org

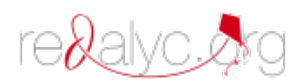

Sistema de Información Científica Red de Revistas Científicas de América Latina, el Caribe, España y Portugal Proyecto académico sin fines de lucro, desarrollado bajo la iniciativa de acceso abierto 


\title{
DESARROLLO FINANCIERO Y CRECIMIENTO ECONÓMICO EN MÉXICO
}

\author{
Domingo Rodríguez Benavides* \\ Francisco López Herrera**
}

Fecha de recepción: 18 de junio de 2009. Fecha de aceptación: 30 de septiembre de 2009.

\begin{abstract}
Resumen
El objetivo del presente trabajo es averiguar si el desarrollo financiero ha influido en el crecimiento económico después de 1990 (el análisis comprende el periodo 1990-2004), lapso en el que se llevó a cabo una serie de reformas que han tendido a liberalizar el sector financiero en México. La hipótesis de McKinon-Shaw establece que las restricciones gubernamentales sobre el sector financiero restringen y distorsionan el proceso de desarrollo de este sector y, por ende, inhiben el proceso de crecimiento económico. La estimación de la incidencia del desarrollo financiero en el crecimiento económico se realiza por medio de una función de producción agregada dinámica, similar a la empleada por Asteriou y Price (2000). En contraposición a los resultados obtenidos por Tinoco, Torres y Venegas (2008), encontramos que el desarrollo financiero ha tenido un impacto positivo en el crecimiento económico en el periodo estudiado.

Palabras clave: desarrollo financiero, crecimiento económico, cointegración, modelos de Corrección del Error, causalidad en el sentido de Granger.
\end{abstract}

* División de Estudios de Posgrado, Facultad de Economía, UNAM. Correo electrónico: domr@economia.unam.mx

** División de Investigación, Facultad de Contaduria y Administración, UNAM. Correo electrónico: francisco_lopez_herrera@yahoo.com.mx

Los autores agradecen los comentarios, críticas y sugerencias de los participantes en el II Foro de Finanzas, Administración de Riesgos e Ingeniería Financiera y en el XIV Congreso Internacional de Contaduría, Administración e Informática, así como de los dictaminadores anónimos. Cualquier error u omisión que prevalezca, es responsabilidad exclusiva de los autores. 


\section{Abstract}

The objective of this study is to ascertain whether financial development influenced economic growth after 1990 (the analysis includes the period 1990 - 2004), a period during which a series of reforms was carried out tending to liberalize Mexico's financial sector. The McKinnon-Shaw hypothesis establishes that government restrictions on the financial sector restrict and distort the process of development in this sector and thereby inhibit the process of economic growth. The estimated incidence of financial development on economic growth is realized using a dynamic aggregate production function, similar to that employed by Asteriou and Price (2000). In contrast with the results obtained by Tinoco, Torres and Venegas (2008), we find that financial development had a positive impact on economic growth in the period under review.

Key words: financial development, economic growth, co-integration, error correction models, Granger-type causality.

\section{Résumé}

L'objectif du présent travail est de vérifier si le développement financier a influé sur la croissance économique après 1990 (l'analyse s'étend de 1990 à 2004), période où ont été menées une série de réformes tendant à libéraliser le secteur financier au Mexique. Selon l'hypothèse de McKinon-Shaw, les restrictions gouvernementales sur le secteur financier restreignent et distorsionnent le processus de développement de ce secteur et, par conséquent, inhibent le processus de croissance économique. L'estimation de l'incidence du développement financier sur la croissance économique est réalisée au moyen d'une fonction de production ajoutée dynamique, similaire à celle employée par Asteriou et Price (2000). Contrairement aux résultats obtenus par Tinoco, Torres et Venegas (2008), nous observons que le développement financier a eu un impact positif sur la croissance économique durant la période étudiée.

Mots clés: développement financier, croissance économique, co-intégration, modèles de correction de l'erreur, causalité dans le sens de Granger.

\section{Resumo}

O objetivo do presente trabalho é averiguar se o desenvolvimento financeiro influiu no crescimento econômico depois de 1990 (a análise compreende o período 1990 - 2004), lapso em que se levaram a cabo uma série de reformas que tendem a liberalizar o setor financeiro no México. A hipótese de McKinon-Shaw estabelece que as restrições governamentais sobre o setor financeiro restringem e distorcem o processo de desenvolvimento deste setor e, em consequência, inibem o processo de crescimento econômico. A estimação da incidência do desenvolvimento financeiro no crescimento econômico se realiza por meio de uma função de produção agregada dinâmica, similar à empregada por Asteriou e Price (2000). Em contraposição aos resultados obtidos por Tinoco, Torres e Venegas (2008), encontramos que o desenvolvimento financeiro tem tido um impacto positivo no crescimento econômico no período estudado.

Palavras-chave: desenvolvimento financeiro, crescimento econômico, cointegração, modelos de Correção do Erro, causalidade no sentido de Granger. 
Introducción

1 vínculo entre desarrollo financiero y crecimiento económico ha recibido considerable atención tanto en el ámbito teórico como en el empírico, principalmente debido a los procesos de liberalización y apertura adoptados en diversos países. En la nueva teoría del crecimiento, la intermediación financiera desempeña una nueva función específica a través del financiamiento de la investigación y desarrollo y la inversión en capital humano, como lo argumentan Tinoco, Torres y Venegas (2008).

Diversos autores han demostrado que el desarrollo del sistema financiero tiene un impacto positivo sobre el crecimiento económico, así como en el volumen y la eficacia de la inversión (véase Fry, 1997). La principal implicación de lo que se conoce como la hipótesis McKinon-Shaw, es que las restricciones gubernamentales sobre el sistema financiero (topes en las tasas de interés, grandes requerimientos de reservas y programas de crédito focalizados o dirigidos) restringen y distorsionan el proceso de desarrollo financiero y, en consecuencia, reducen el crecimiento de la economía. Por ello se ha denominado estado de represión financiera a la situación en la que tales restricciones están presentes en el funcionamiento del sistema financiero. Este efecto negativo sobre la economía se debe a las imperfecciones que provocan esas restricciones en los mercados financieros, produciendo una asignación ineficiente de recursos.

Los estudios sobre el crecimiento endógeno que incorporan tanto al crecimiento como a las instituciones financieras en forma endógena, muestran resultados similares. No obstante, de acuerdo con Andriesz, Asteriou y Pilbeam (2003), en este enfoque no se establece claramente la dirección de la causalidad entre desarrollo financiero y crecimiento económico. En estos modelos, la intermediación financiera se configura explícitamente y se sugiere que tiene un efecto positivo sobre el crecimiento económico; en este sentido, Greenwood y Jovanovic (1990) y King y Levine (1993b) argumentan que la intervención gubernamental en el sistema bancario reduce la tasa de crecimiento de la economía. Por otra parte, pocas pero crecientes investigaciones, de autores como Van Winjbergen (1983) y Stiglitz (1994), señalan que las imperfecciones del mercado financiero, tales como información asimétrica y competencia imperfecta, pueden tener un efecto negativo sobre el crecimiento económico.

En términos generales, la literatura sobre el tema se puede clasificar en tres grandes visiones sobre la importancia del sector financiero en el crecimiento económico. La primera considera al sector financiero como un elemento fundamental para el crecimiento (Schumpeter, 1911; Goldsmith, 1969; McKinnon, 1973; Shaw, 1973; 
Odedokun, 1996; King y Levine, 1993a y 1993b). También hay quienes adoptan una posición diamentralmente opuesta pues ven a dicho sector como un factor sin mucha importancia relativa para el crecimiento (Robinson, 1952; Lucas, 1988; y Stern, 1989). La tercera visión se concentra en el impacto potencialmente negativo del funcionamiento del sector financiero sobre el crecimiento (Van Wijngergen, 1983; Buffie, 1984; entre otros). En forma paralela a esas visiones, se ha llevado a cabo una cantidad sustantiva de estudios empíricos; sin embargo, algunos de ellos han encontrado evidencia mixta respecto de los efectos del desarrollo financiero sobre el crecimiento económico: a diferencia de lo que se esperaría según la hipótesis McKinon-Shaw, hay casos en que no se observa una relación positiva, mientras que en otros análisis ni siquiera se detecta una relación significativa, como lo destaca Xu (2000).

No obstante el gran interés suscitado en torno a las relaciones entre el desarrollo de los sistemas financieros y el crecimiento de la economía, algunas cuestiones no están del todo claras en el análisis teórico y empírico de este importante vínculo; por ejemplo, destaca el hecho de que no se sabe con precisión si los mercados bursátiles liberalizados han resultado realmente importantes para el crecimiento económico.

Pese al avance mostrado por los desarrollos teóricos en esta área, éstos tampoco establecen de forma indubitable la dirección de la causalidad entre desarrollo financiero y crecimiento económico. Tal es el caso de McKinnon (1988), quien se cuestiona: “¿Cuál es la causa y cuál es el efecto? ¿Es el sector financiero un promotor del desarrollo económico, o simplemente sigue al crecimiento del producto real que es generado por otros factores?" Hasta la fecha no existe consenso respecto de que el desarrollo financiero determine el crecimiento económico, o si sucede lo contrario, o bien si ambos procesos se determinan de manera recíproca. Recientemente, una serie de trabajos empíricos han intentado establecer la dirección de la causalidad entre desarrollo financiero y crecimiento económico. Algunos tienden a confirmar una asociación positiva entre el desarrollo financiero y el crecimiento económico.

En este trabajo tratamos la cuestión de la causalidad entre el desarrollo financiero y el crecimiento económico usando datos de series de tiempo para México. Nuestro objetivo es determinar en primera instancia si en México existe una relación de largo plazo entre el crecimiento económico y el desarrollo financiero; asimismo, buscamos evidencia econométrica sobre el posible sentido de la causalidad. Por tales motivos, mediante el análisis de cointegración y de causalidad en el sentido de Granger se estudian las relaciones entre el PIB per cápita real, un indicador de desarrollo financiero, y la relación capital-trabajo. Es decir, en el diseño para este análisis aproximamos una función de producción agregada con la cual tratamos de incorporar la dinámica del 
sector real de la economía para explicar, junto con el desarrollo financiero, el crecimiento de la economía mexicana.

El artículo está estructurado de la siguiente forma: primero, se ofrece una breve revisión de una muestra relevante de trabajos empíricos sobre el tema; después, se brinda una panorámica general de los antecedentes que condujeron al proceso de reforma económico-financiera en México; posteriormente se describen los datos y la metodología econométrica que sustentan el análisis empírico, cuyos resultados se muestran a continuación; para finalizar, se presentan las conclusiones.

\section{Revisión de la literatura empírica}

El trabajo de Goldsmith (1969) es considerado el estudio seminal sobre el análisis de la relación entre crecimiento económico y algunas medidas agregadas que dan cuenta del desempeño del sistema financiero. Goldsmith analiza la correlación entre el crecimiento económico y el desarrollo de la intermediación financiera en 35 países, basándose en los datos disponibles de 1860 a 1963. Los resultados de ese estudio pionero sugieren, en términos generales: $a$ ) el desempeño económico está asociado positivamente al desarrollo financiero, y $b$ ) los periodos de más rápido crecimiento económico suelen estar acompañados de una tasa de desarrollo financiero superior a la promedio.

Levine (1995) estudia la relación entre el desarrollo financiero y el crecimiento económico de 80 países, analizando diversos indicadores de desarrollo financiero con técnicas de regresión en sección cruzada; sus resultados muestran que los países con sistemas financieros mayores en 1960 crecieron más rápidamente en los siguientes 30 años que aquellos países con sistemas financieros inicialmente pequeños. Estos resultados apuntan a que la relación entre ambas variables es positiva y que el crecimiento de la economía puede verse favorecido en la medida en que los sistemas financieros se encuentren desarrollados.

Asteriou y Price (2000) examinan el vínculo entre desarrollo financiero y crecimiento económico a través de la estimación de una función de producción agregada, en la que relacionan el PIB per cápita en términos reales, la razón M2/PIB a nivel agregado, como indicador agregado de desempeño económico, y la razón capital-trabajo. A través de las pruebas de cointegración y de causalidad, ellos encuentran evidencia a favor de la hipótesis de que el desarrollo financiero auspicia el crecimiento económico para el caso del Reino Unido.

Vol. 40, núm. 159, octubre-diciembre / 2009 
Romero de Ávila (2003) estudia el caso europeo valiéndose de técnicas de datos en panel que permiten controlar tanto los factores omitidos a través de la inclusión de efectos específicos para cada país, como los efectos del ciclo económico a través de variables dummies en el tiempo. Sus resultados sugieren que en el caso europeo la adopción de medidas destinadas a favorecer el desarrollo del sistema financiero, apoyando la integración de los mercados y el aumento de la competencia, es capaz de favorecer el crecimiento económico. Romero de Ávila sostiene que el impacto de la supresión de los controles sobre los movimientos de capital alcanzaría al menos a una quinta parte de la tasa de crecimiento medio del PIB registrada durante el periodo 1960-2001, mientras que la armonización normativa habría aportado al PIB un aumento anual de casi uno por ciento.

Bhattacharya y Sivasubramaniam (2003) analizan el caso de la India durante 1970-1999, utilizando las técnicas de raíces unitarias y cointegración. En su modelo incorporan únicamente las tasas de crecimiento del PIB y de la razón M3 a PIB; sus resultados tienden a favorecer la hipótesis de que el desarrollo financiero incentiva significativamente el incremento de las actividades productivas.

Dritsakis y Adamopoulos (2004) utilizan la metodología de los vectores autorregresivos para analizar la relación entre el desarrollo financiero de Grecia y su crecimiento económico durante el periodo muestral 1960-2000. Utilizando datos trimestrales para las tasas de crecimiento del PIB, las razones M2 a PIB, como proxy para el desarrollo financiero, y la apertura comercial a PIB, encuentran un vector de cointegración entre esas variables y una causalidad bidireccional entre desarrollo financiero y crecimiento económico. Es decir, se establece que entre el desarrollo financiero griego y el crecimiento de esa economía existe una relación de largo plazo que es estadísticamente significativa; sin embargo, estos resultados no permiten sustentar que el desarrollo de su sistema financiero sea la causa del crecimiento de la economía en Grecia.

Christopoulos y Tsionas (2004) estudian 10 países en vías de desarrollo empleando pruebas de cointegración de umbral y un modelo de corrección del error (MCE) de panel, con la finalidad de probar dos hipótesis: 1) la existencia de un equilibrio único entre finanzas, crecimiento y otras variables de control, y 2) que la dirección de la causalidad va del desarrollo financiero hacia el crecimiento económico, y no a la inversa. Los resultados de su estudio verifican ambas hipótesis.

Ruiz (2004), mediante un procedimiento de doble técnica econométrica que involucra regresiones de MCO y modelos SERSRA de Zellner-Schmidt, investiga la importancia del desarrollo de los mercados financieros locales en el desarrollo económico, y evalúa los efectos de externalidades entre las economías de Argentina, Brasil, Chile, 
Colombia y México mediante datos anuales de 1948 a 1998, usando la estructura de crecimiento endógeno. Al comparar sus resultados con los reportados por Murinde (1996), Ruiz interpreta la existencia de efectos diferenciados en la vinculación entre mercados financieros y crecimiento económico como sugerentes de que algunos mercados financieros específicos son los que pueden desempeñar una función importante en el crecimiento.

En su estudio, Abu-Bader y Abu-Qarn (2005), valiéndose de la prueba de causalidad de Granger, encuentran evidencia significativa de que el desarrollo financiero de Egipto contribuye a explicar el crecimiento económico observado en ese país de 1960 a 2001. En Abu-Bader y Abu-Qarn (2006) se extiende el análisis a la relación causal entre el desarrollo financiero local y el crecimiento económico de Argelia, Egipto, Marruecos, Siria y Túnez durante el periodo 1960-2004. Sin embargo, sirviéndose también de pruebas de causalidad de Granger, encuentran únicamente un apoyo estadísticamente débil para sustentar tanto la existencia de una relación de largo plazo entre desarrollo financiero y crecimiento económico como la hipótesis de que el desarrollo financiero conduce al crecimiento. Es de señalarse que en los casos en que detectaron cointegración, la causalidad se dirige del crecimiento económico al desarrollo financiero o la causalidad es bidireccional para este conjunto de países.

En un estudio más reciente, Tinoco, Torres y Venegas (2008) investigan si el desarrollo financiero de largo plazo en México ha incidido de manera positiva sobre la actividad productiva. Para tal fin, ellos utilizan una función de producción dinámica agregada para examinar dos posibles efectos: 1) la regulación financiera puede tener un efecto negativo sobre el crecimiento económico, y 2) la intermediación financiera tiene un impacto positivo sobre la actividad productiva. Recurren a dos especificaciones de la función de producción dinámica agregada, cuyas variables son el PIB y la formación de capital fijo, las cuales combinan con un indicador de desarrollo financiero en la primera especificación y con un índice de represión financiera en la segunda. La construcción de estos indicadores se realizó a través de la técnica del análisis de componentes principales. En su análisis también emplean pruebas de causalidad en un modelo de corrección del error, encontrando que ni el indicador de desarrollo financiero ni la regulación financiera afectan la dinámica del PIB; no obstante, muestran evidencia de que la regulación tiene un efecto negativo sobre el desarrollo financiero.

En síntesis, el vínculo entre desarrollo financiero y crecimiento económico se ha analizado desde diferentes perspectivas y a través de distintas técnicas econométricas como son: análisis de regresión, las técnicas de series de tiempo y los métodos de 
panel de datos, principalmente. La elección de la técnica a emplear por lo general ha estado sujeta al objetivo que persigue(n) el autor (los autores) y del alcance propuesto para el estudio, un país en particular o bien para un conjunto de países. Una de las ventajas de utilizar las técnicas de series de tiempo como las pruebas de raíces unitarias y de cointegración a través de un MCE como el que empleamos en el presente estudio, y cuyos resultados se reportan en páginas posteriores, es que permiten responder a cuestiones tales como la dirección de la causalidad entre el indicador de desarrollo financiero y el crecimiento económico, cuya determinación en el caso mexicano es nuestro principal objetivo.

\section{Antecedentes de la reforma financiera mexicana}

A principios de la década de 1980, las autoridades económicas en México emprendieron una serie de ajustes y reformas con la finalidad de estabilizar la economía, ya que ésta se encontraba sumida en una profunda crisis, exacerbada por las dificultades para el acceso al financiamiento proveniente de los circuitos del crédito internacional. En 1988 se profundizaron estos cambios en la estrategia de desarrollo interno, tratando de acelerar la reforma estructural iniciada unos años antes. De esta manera se buscó acentuar la importancia del sector exportador, restringir el papel hasta entonces preponderante del sector público y sentar las bases para que el sector privado se convirtiera en el principal motor de la economía (Solís, 1996).

Antes de iniciar tales reformas, el Estado mexicano era el principal impulsor de la economía, pues su participación activa en los sectores más importantes de la producción de bienes e incluso de algunos servicios había venido creciendo a tasas cada vez más aceleradas. Según las estadísticas disponibles, a esta fuerte participación estatal en las principales ramas de actividad económica se asociaron altas tasas de crecimiento del PIB, las cuales se sostuvieron por varias décadas llegando incluso a niveles que no se han visto nuevamente, por lo menos en los últimos 25 años.

El gasto público era el motor del crecimiento económico y social, por lo que de manera un tanto natural, la participación del sector público de México en la actividad económica estaba acompañada de un nivel del gasto público en constante crecimiento (Tello, 2007). Para cubrir los crecientes déficit asociados a la expansión del gasto público, el gobierno mexicano tuvo que recurrir al endeudamiento externo como complemento, también en una escala creciente.

El objetivo primordial del endeudamiento de la economía mexicana era complementar el financiamiento que podía obtenerse en el mercado financiero interno, ya que como éste se encontraba reprimido, ofrecía una forma barata y no inflacionaria 
de financiar el déficit presupuestal (Aspe, 1993). En el caso de México, esa represión financiera se manifestaba como un conjunto de medidas tales como la regulación de las tasas de interés (tanto activas como pasivas), la existencia de controles cuantitativos del crédito, el uso de reservas obligatorias por parte de la banca comercial para el otorgamiento de crédito al gobierno, etc. Durante el sexenio del presidente Carlos Salinas (1989-1994), las autoridades económicas argumentaban que la reforma financiera era necesaria, indispensable e improrrogable debido a que bajo el esquema de operación en que había funcionado la economía del país, se había desarrollado un marco institucional incapaz de responder a los choques externos y a los efectos desfavorables de éstos sobre los recursos disponibles para financiar las actividades productivas.

También en el exterior se produjeron condiciones que dieron pie a la búsqueda de cambios en el funcionamiento económico y su relación con los circuitos de financiamiento. El estancamiento económico y la inflación (estanflación) observados en los países desarrollados a finales de los setenta indujeron altas tasas de interés, que aunadas a la caída de los precios petroleros ocasionaron que el gobierno mexicano enfrentase problemas para servir la deuda contraída en los circuitos internacionales de crédito. Lo mismo sucedió a otros países relativamente pobres y altamente endeudados, por lo que a tal situación se le denominó problema de la deuda de los países del Tercer Mundo. Para México, la consecuencia inmediata de ese problema fue la restricción al crecimiento durante la década siguiente; los nuevos endeudamientos que se negociaban cada vez con mayor dificultad sólo servían para pagar los viejos empréstitos y los recursos obtenidos no se canalizaban a la actividad productiva. En consecuencia, el gobierno mexicano emprendió la reforma económica: transfirió a la iniciativa privada la tarea de impulsar la economía, incluyendo el financiamiento para las actividades productivas mediante el nuevo marco regulatorio de liberalización y apertura del sistema financiero. En términos del discurso oficial, la expectativa era promover el crecimiento económico a través del desarrollo financiero.

En el contexto macroeconómico nacional, con el argumento de constituir medios suficientes de financiamiento para las actividades productivas de las empresas, el inicio de la década de 1990 se caracterizó fundamentalmente por el proceso de liberalización del sistema financiero mexicano. Dentro del conjunto de reformas impulsadas por el gobierno, destaca la liberalización de la banca efectuada entre 1988 y 1991. Las medidas de política para alcanzar esa meta incluían la eliminación de los requerimientos de reservas por parte de los bancos privados y la supresión de las restricciones a las tasas de interés, tanto de los depósitos como de los préstamos. La reprivatización de 18 bancos entre 1991 y 1992 auspició la conformación de nuevos y poderosos grupos finan- 
cieros, con un alto grado de concentración, pues esas nuevas instituciones financieras controlaban más de 50\% de los activos en 1994 (Tinoco, Torres y Venegas, 2008).

La modernización de la estructura financiera tenía como sustento la internacionalización del sistema financiero frente al inminente proceso de globalización. Se creó un nuevo marco jurídico que autorizaba a los intermediarios financieros mexicanos, ahora nuevamente privados, a llevar a cabo actividades en los mercados de capitales internacionales; también se autorizó a que grandes grupos financieros extranjeros iniciaran operaciones en México.

En pocas palabras, para el proceso de reforma de la economía mexicana se conformaron nuevos marcos legales para regular el funcionamiento del sistema financiero nacional, cuyas principales consecuencias fueron el funcionamiento de mercados liberados de la mediación gubernamental, tipo de cambio flotante dentro de bandas (posteriormente bajo régimen de libre flotación), no fijación de las tasas de interés pasivas ni activas y liberalización del flujo de movimientos de capitales con los mercados financieros internacionales.

A principios de los noventa, por fin parecía alcanzarse la meta de estabilizar la economía; sin embargo, la gran cantidad de choques negativos externos e internos al inicio de esa década culminó con la crisis financiera y de balanza de pagos que estalló a finales de 1994. Dado el agotamiento relativamente acelerado de las reservas internacionales que se dio desde principios de 1994, el Banco Central fue incapaz de mantener la paridad cambiaria, por lo que a finales de ese año las autoridades mexicanas tomaron la decisión de abandonar el régimen de tipo de cambio semifijo dentro de "bandas" que se había seguido y optaron por un nuevo régimen cambiario de tipo flotante, de acuerdo con las fuerzas del mercado. Para inicios de 1995, el panorama era completamente negativo ante las expectativas generalizadas de una nueva recesión y el franco repudio a los activos financieros mexicanos, públicos y privados, por parte de los inversionistas internacionales que, cuando las expectativas eran optimistas, los habían adquirido de muy buena gana.

Se ha argumentado que las políticas económicas que se instrumentaron como consecuencia de la crisis tuvieron éxito en la estabilización de la macroeconomía, debido, entre otras cosas, a los diversos paquetes negociados con el FMI y con el gobierno de los Estados Unidos para rescatar a las empresas internas con insolvencia y a los deudores de la banca. Ya superada la crisis financiera de 1994-1995, el gobierno comenzó a reestructurar el sistema bancario y a rescatar a los deudores de la banca, lo que generó el incremento intempestivo de las tasas de interés (Tinoco, Torres y Venegas, 2008). Adicionalmente, las autoridades establecieron un nuevo marco de estricta supervisión y regulación preventivas que se basaba en la autorregulación, 
también crearon un nuevo sistema de pensiones basado en el funcionamiento de fondos privados para fortalecer el ahorro interno, y permitieron la inversión extranjera en la banca comercial doméstica.

\section{Datos y metodología econométrica}

Siguiendo la especificación empleada por Asteriou y Price (2000), en el presente estudio se estima una función de producción agregada dinámica en la cual la variable independiente es un indicador de crecimiento económico; en este caso se tomó el PIB real per cápita, el cual se ha empleado en diversos estudios empíricos sobre el tema, por ejemplo Roubini y Sala-i-Martín (1992) y King y Levine (1993a; 1993b). La función de producción especificada es la siguiente:

$$
\frac{p i b r}{p o b}=f\left(\frac{m 4}{\text { pibn }}, \frac{k}{l}\right)
$$

Donde $p i b r$ / pob es el PIB per cápita en términos reales; $m 4$ / pibn es la razón M4 a PIB nominal, ambas variables en términos nominales, lo que comúnmente se emplea para medir el grado de desarrollo financiero a nivel agregado, y $k / l$ es la razón capitaltrabajo. Se espera, a priori, que cada una de las variables incida de manera positiva en el PIB per cápita. Se eligió el PIB per cápita en términos reales y no la productividad laboral agregada como indicador del crecimiento económico debido a que este tipo de modelos se basan en la especificación convencional de la ecuación del crecimiento que se define a partir de la siguiente función (Barro y Sala-i-Martín, 1995):

$$
Y_{t}=F\left(A, K_{t}, L_{t}\right)
$$

Donde $K_{t}$ y $L_{t}$ son el capital y el trabajo, respectivamente, mientras que el factor $A$ se asocia a algún índice de conocimientos o al nivel de la tecnología que, a diferencia del modelo de crecimiento neoclásico tradicional, no se asume como exógeno. De esta forma, si se supone que la función de producción tiene la forma de una función CobbDouglas, entonces es posible estimar la incidencia de los factores de la producción en el crecimiento económico.

En estudios empíricos sobre modelos de crecimiento, como Ross (2000) y Bleaney y Nishiyama (2002), se utiliza comúnmente como variable dependiente al producto per cápita, la cual es el objetivo a explicar, ya que normalizan a la ecuación (2) por medio de la variable empleo, y de esta manera se requiere asumir que la tasa de crecimiento de la fuerza laboral corresponde a la tasa de crecimiento poblacional. Los datos sobre el capital agregado se obtuvieron de las estimaciones de acervos de capital realizadas por

Vol. 40, núm. 159, octubre-diciembre / 2009

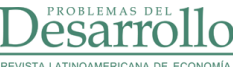


Loría y De Jesús (2007). Como variable representativa del empleo agregado se tomó el número de trabajadores registrados en el IMSS. En cuanto a la población nacional, debido a que en las estadísticas oficiales sólo se cuenta con información histórica de la población total por año, fue necesario trimestralizar esta variable asumiendo una tasa de crecimiento constante a lo largo de cada año ${ }^{1}$. Los datos de esta variable junto con otras empleadas en la estimación se encuentran en el cuadro 1 (véase apéndice).

Por otra parte, la literatura existente sugiere como proxy para el desarrollo financiero la razón de una medida amplia de dinero (generalmente M2 o M4) al PIB en términos nominales. De acuerdo con algunos autores, esta razón mide la magnitud de la monetización, en lugar de la profundización financiera. En nuestro análisis empleamos como indicador de desarrollo financiero la razón M4 a PIB en términos nominales. Esta variable está diseñada para mostrar el tamaño real del sector financiero (Asteriou y Price, 2000), la razón se incrementa (o se reduce) en el tiempo si el desarrollo del sector financiero se acelera (o desacelera) en comparación con el sector real de la economía.

Las gráficas 1, 2 y 3 presentan la evolución de las series del PIB per cápita, de la razón M4 a PIB y de la razón capital-trabajo, respectivamente.

\section{Gráfica 1}

Logaritmo natural de PIB per capita

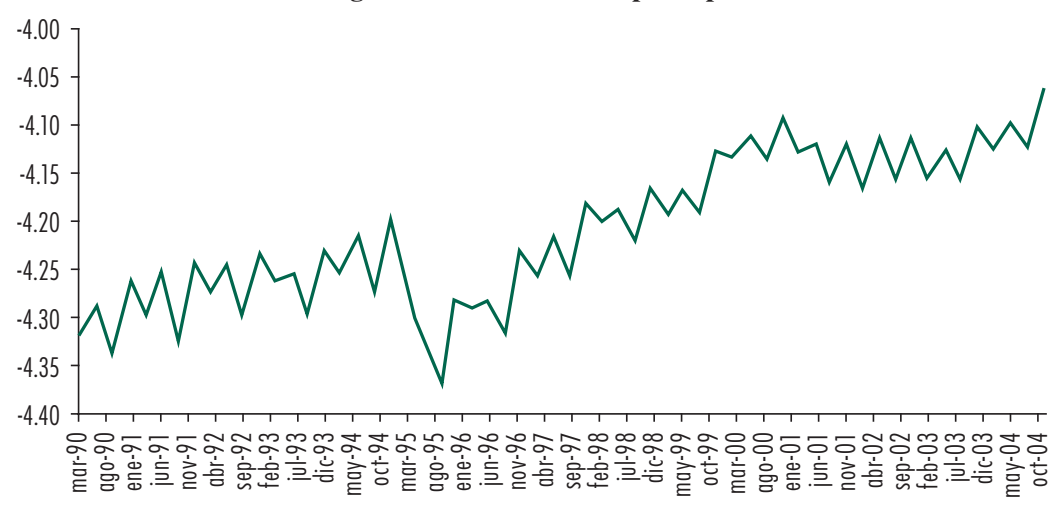

1 Este supuesto empleado en la construcción de la serie trimestral de la población total puede no ser muy burdo puesto que no hay razones para considerar grandes fluctuaciones en el crecimiento poblacional; de hecho, la tendencia encontrada en la tasa de crecimiento anual de la población es ligeramente decreciente en el periodo estudiado. 
Gráfica 2

Logaritmo natural de la razón m4/pibn

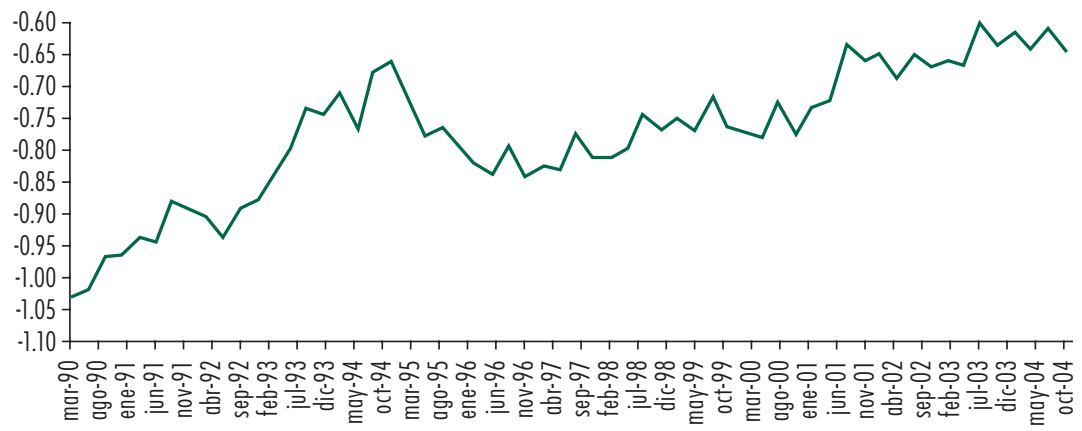

Gráfica 3

Logaritmo natural de la razón $k / l$

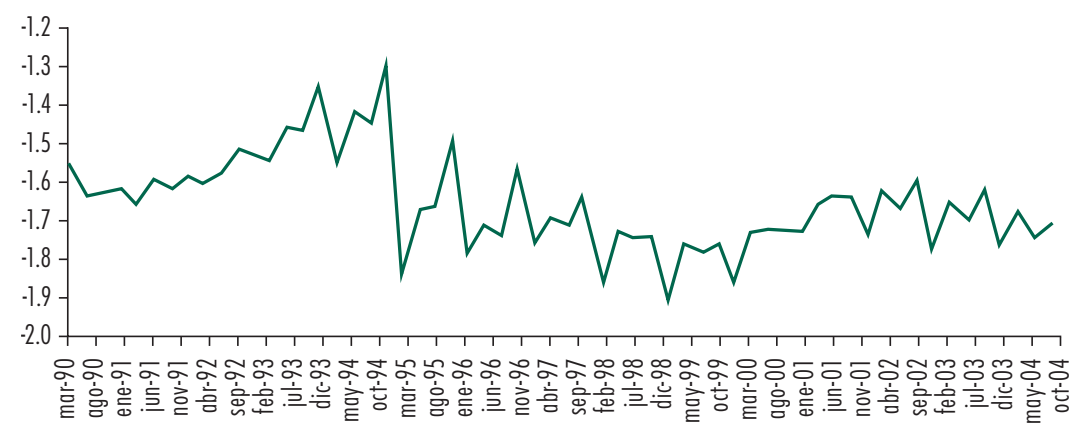

Como se puede apreciar en las gráficas, las series presentan ruptura en el primer trimestre de 1995, a causa de la crisis económico-financiera que se suscitó en ese año, la cual es más notoria tanto en el PIB per cápita en términos reales como en la razón capital-trabajo. De igual forma es posible identificar un ligero quiebre en el primer trimestre de las series, en el cual cambian levemente su tendencia.

Con el fin de identificar si las series incorporadas en (1) se comportan como caminatas aleatorias, se ejecutaron pruebas de raíces unitarias. De acuerdo con la prueba Dickey-Fuller Aumentada (ADF), la hipótesis de una raíz unitaria se constata a través de la estimación del siguiente modelo:

$$
\Delta y_{t}=\alpha_{0}+\gamma y_{t-1}+\sum_{i=2}^{p} \beta_{i} \Delta y_{t-i+1}+\varepsilon_{t}
$$


En la especificación anterior, el interés radica en probar la hipótesis nula de $\gamma=0$. Si $\gamma=0$ entonces se dice que la serie $y_{t}$ tiene una raíz unitaria.

Por otra parte, la técnica empleada para probar cointegración entre las series fue llevada a cabo empleando el método de Johansen, que prueba el rango de $\Gamma_{k}$, la matriz de parámetros asociada al vector de rezagos en los niveles de las variables. Esta técnica especifica el modelo de corrección del error (MCE) de un VAR de $m$-variables para un vector de series de tiempo $X_{t}$ como:

$$
\Delta X_{t}=\Gamma_{1} \Delta X_{t-1}+\Gamma_{2} \Delta X_{t-2} \ldots+\Gamma_{k-1} \Delta X_{t-k+1}+\Gamma_{k} X_{t-k}+v_{t}
$$

donde $\Gamma_{k}$ define la "solución en niveles" de largo plazo en la ecuación (4) (véase Cuthbertson, 1992), y $k$ es lo suficientemente grande para asegurar que $v_{t}$ sea un vector de ruido blanco gaussiano que se distribuye idéntica e independientemente con media cero y varianza finita. De esta forma, la técnica prueba el rango de $\Gamma_{k}$, la matriz de parámetros asociada al vector de rezagos en los niveles de las variables.

\section{Análisis empírico}

Con el fin de averiguar el orden de integración de las series consideradas en el modelo, se realizaron pruebas Dickey-Fuller Aumentadas (ADF) para verificar la posible presencia de raíces unitarias. Las series del PIB per cápita en términos reales y de la razón capital-trabajo rechazan la hipótesis nula de la raíz unitaria al nivel de significancia de $5 \%$ pero no a $1 \%$, mientras que para la serie de $m 4 /$ pibn no se puede descartar la hipótesis nula de la raíz unitaria para ambos niveles de significancia. Sin embargo, las tres series revelaron estacionariedad en sus primeras diferencias, como se puede apreciar en la tabla 1. Las pruebas se llevaron a cabo utilizando la metodología de lo general a lo particular. No obstante estos resultados, para el análisis que sigue se asume que las series consideradas poseen una raíz unitaria en los logaritmos naturales de sus niveles.

La prueba de cointegración que se llevó a cabo es la de Mosconi, Johansen y Nielsen (2000), la cual es una variante de (4) que permite incorporar rupturas en la series. Dicha prueba se especificó asumiendo dos rupturas estructurales en las series por su comportamiento histórico observable, la primera en el trimestre 1995-1 y la segunda en el trimestre 2000-1. En la parte determinista se incorporaron dummies estacionales en la forma estándar. La longitud de los rezagos fue elegida de acuerdo con el Criterio de Schwarz y los resultados de la prueba se presentan en la tabla 2. 
Tabla 1

Pruebas Dickey-Fuller Aumentada para las series 1990:1-2004:4

\begin{tabular}{|l|c|c|c|c|c|}
\hline \multicolumn{1}{|c|}{ Serie } & $t_{a 1}$ & $t_{\gamma l}$ & Rezagos & $5 \%$ & $1 \%$ \\
\hline$(\text { pibr } / \text { pob })_{t}$ & $-3.669^{\star}$ & 10.615 & 4 & -3.494 & -4.134 \\
\hline$\Delta(\text { pibr } / \text { pob })_{t}$ & $-5.902^{\star *}$ & 7.160 & 4 & -1.947 & -2.608 \\
\hline$(m 4 / p i b n)_{t}$ & -3.380 & 5.554 & 4 & -3.494 & -4.134 \\
\hline$\Delta(m 4 / p i b n)_{t}$ & $-9.243^{\star \star}$ & -4.627 & 3 & -7.947 & -2.608 \\
\hline$(k / l)_{t}$ & $-2.923^{\star}$ & 8.230 & 4 & -2.916 & -3.555 \\
\hline$\Delta(k / l)_{t}$ & $-10.645^{\star *}$ & -7.800 & 3 & -7.947 & -2.608 \\
\hline
\end{tabular}

Nota: $t_{a 1}$ representa el $t$-estadístico de la prueba Dickey-Fuller; $t_{\gamma l}$ el $t$-estadístico del último rezago. Pruebas realizadas en Eviews 5.1.

Tabla 2

Resultados de la Prueba de la Traza de Johansen

\begin{tabular}{|c|c|c|c|c|c|c|}
\hline Periodo & $H_{0}:$ rango $=p$ & $-T \sum_{i=r+1}^{p} \ln \left(1-\hat{\lambda_{r+1}}\right)$ & Valor- $p$ & $90 \%$ & $95 \%$ & $99 \%$ \\
\hline 1990:1-2004:4 & $p=0$ & $75.29^{\star *}$ & 0.0000 & 46.84 & 49.75 & 55.50 \\
\hline & $p \leq 1$ & $35.48^{\star}$ & 0.0124 & 28.80 & 31.27 & 36.08 \\
\hline & $p \geq 2$ & 14.27 & 0.1000 & 14.27 & 16.25 & 20.43 \\
\hline
\end{tabular}

Nota: *** indican el rechazo de la hipótesis nula a $5 \%$ y a $1 \%$ de significancia, respectivamente. Pruebas realizadas en $J$-Multi 4.23 .

La prueba de la traza indica al menos una ecuación cointegrante al nivel de significancia de cinco por ciento.

Una vez que se obtuvo evidencia sobre la existencia de cointegración entre las variables, estimamos el modelo de corrección del error (MCE) en el cual se incorporaron tanto dummies estacionales centradas como una dummy de impulso -arriba descrita-, y que da cuenta de las rupturas de las series incluidas en la prueba de cointegración de Mosconi, Johansen y Nielsen (2000). El vector cointegrante estimado a través de la técnica de Johansen se muestra en la tabla 3.

Tabla 3. Vector Cointegrante Estandarizado para

$$
\beta_{0}+\beta_{1}(m 2 / p i b n)_{t}+\beta_{2}(k / l)_{t}
$$

\begin{tabular}{|c|c|c|}
\hline$\beta_{0}$ & $\beta_{1}$ & $\beta_{2}$ \\
\hline 4.582 & 0.524 & -0.470 \\
\hline$(64.271)$ & $(14.231)$ & $(-13.677)$ \\
\hline
\end{tabular}

Nota: Los números entre paréntesis son los estadísticos $t$. Estimaciones realizadas en $J$-Multi 4.23. 
De acuerdo con los coeficientes del vector cointegrante, el desarrollo financiero impacta de manera positiva sobre el crecimiento económico, pero en una proporción menor a la unidad. Sin embargo, al contrario de lo que se esperaba, la relación capitaltrabajo impacta en forma negativa sobre el crecimiento económico, resultado que quizá pueda atribuirse a la correlación negativa que muestra la razón capital-trabajo tanto con el PIB per cápita como con el indicador de desarrollo financiero a partir de 1995, como se muestra en las gráficas 1, 2 y 3 . De esta manera, el signo del coeficiente estimado para la relación capital-trabajo para México no es consistente con los resultados de Asteriou y Price para el Reino Unido.

Por su parte, los coeficientes alpha que miden la velocidad de ajuste del término de corrección de error en el MCE, permiten realizar pruebas de exogeneidad débil, las cuales se muestran en la tabla 4.

Tabla 4

Coeficientes Alpha del MCE

\begin{tabular}{|c|c|c|}
\hline$\Delta(\mathrm{pibr} / \mathrm{pob})_{t}$ & $\Delta(\mathrm{m} 4 / \mathrm{pibn})_{t}$ & $\Delta(\mathrm{k} / \mathrm{l})_{t}$ \\
\hline-0.429 & 0.339 & -0.330 \\
\hline$(-5.126)$ & $(2.874)$ & $(-0.834)$ \\
\hline
\end{tabular}

Nota: Los números entre paréntesis son los estadísticos t.

Como se puede apreciar en esta tabla, los coeficientes que resultaron significativos fueron los del PIB per cápita y el indicador del desarrollo financiero, sin embargo, para que dicha inferencia sea válida se requiere, de acuerdo con la econometría dinámica, que el signo de dicho coeficiente sea negativo y menor a la unidad; los únicos coeficientes de velocidad de ajuste que cumplen con estas condiciones en el modelo de corrección son los que corresponden a la ecuación del PIB per cápita real y a la relación capital-trabajo, aunque este último no resultó estadísticamente significativo. Así, es posible inferir que la única variable que resultó exógena débilmente fue la relación capital-trabajo.

Las tablas 5 y 6 muestran los resultados de la prueba de normalidad, y la tabla 7 presenta los resultados de las pruebas de autocorrelación y de heterocedasticidad del modelo. Como se puede apreciar en dichas tablas, los residuos cumplen con los supuestos de normalidad, con excepción de que el modelo no supera la prueba de curtosis de Doornik y Hansen (1994) a 5\%; tampoco la ecuación 3 supera la prueba de Jarque Bera. Este último resultado no es un problema puesto que esta variable resultó ser exógena débil en el MCE, de igual forma las pruebas LM tampoco revelan 
Tabla 5

Pruebas de normalidad para los residuos del MCE

\begin{tabular}{|l|c|c|c|c|}
\hline & \multicolumn{2}{|c|}{ Doornik y Hansen (1994) } & \multicolumn{2}{c|}{ Lütkepohl (1993) } \\
\hline Prueba & Estadístico & Probabilidad & Estadístico & Probabilidad \\
\hline Conjunta & 9.6015 & {$[0.1425]$} & 1.4606 & {$[0.9621]$} \\
\hline Asimetría & 0.3233 & {$[0.9556]$} & 1.3183 & {$[0.7248]$} \\
\hline Curtosis & 9.2782 & {$[0.0258]$} & 0.1423 & {$[0.9863]$} \\
\hline
\end{tabular}

Nota: Pruebas realizadas en J-Multi 4.23.

Tabla 6

Pruebas de normalidad Jarque-Bera para los residuos del MCE

\begin{tabular}{|c|c|c|c|c|}
\hline Variables & Estadístico-t & Valor $-p$ de $\chi^{2}$ & Asimetría & Curtosis \\
\hline$\Delta(p i b r / p o b)_{t}$ & 0.5768 & {$[0.7495]$} & -0.2456 & 2.8774 \\
\hline$\Delta(m 4 / p i b n)_{t}$ & 0.0163 & {$[0.9919]$} & -0.0419 & 2.9862 \\
\hline$\Delta(k / l)_{t}$ & 13.9497 & {$[0.0009]$} & -0.0635 & 5.4867 \\
\hline
\end{tabular}

Nota: Pruebas realizadas en J-Multi 4.23.

Tabla 7

Pruebas de diagnóstico del MCE de manera conjunta

\begin{tabular}{|c|c|c|}
\hline Prueba & Estadístico & Probabilidad \\
\hline Autocorrelación & & \\
\hline$L M(1)$ & 7.6994 & {$[0.5647]$} \\
\hline$L M(2)$ & 18.6386 & {$[0.4144]$} \\
\hline$L M(4)$ & 49.0171 & {$[0.0726]$} \\
\hline$L M(5)$ & 60.1283 & {$[0.0651]$} \\
\hline Heteroscedasticidad & & {$[0.5552]$} \\
\hline ARCH - LM (1) & 38.1826 & {$[0.6002]$} \\
\hline ARCH - LM (4) & 139.0797 & \\
\hline
\end{tabular}

Nota: Los números entre paréntesis son los rezagos incorporados en cada prueba.

la presencia de autocorrelación y los residuos son homoscedásticos. El hecho de que el modelo cumpla en su mayoría con estos supuestos, permite realizar inferencia económica válida con base en el MCE.

Por último, la tabla 8 muestra los resultados de la prueba de no causalidad en el sentido de Granger en el MCE; como se aprecia, se rechazan ampliamente las hipótesis nulas de no causalidad en ambos sentidos entre las variables indicador de desarrollo financiero y crecimiento económico. 
Tabla 8

Pruebas de No Causalidad en el sentido de Granger

\begin{tabular}{|c|c|c|}
\hline Hipótesis nula & $\chi^{2}(5)=$ & valor $-p$ \\
\hline$m 4 /$ pibn no causa a pibr $/$ pob & $22.2087^{\star \star}$ & 0.0005 \\
\hline $\mathrm{pibr} /$ pob no causa a $4 \mathrm{~m} /$ pibn & $19.3225^{\star \star}$ & 0.0017 \\
\hline
\end{tabular}

Nota: ** indica el rechazo de la hipótesis nula $1 \%$ de significancia.

En nuestro modelo estimado encontramos evidencia a favor de la determinación simultánea entre estas variables, resultado similar al de Dritsakis y Adamopoulos (2004), para Grecia, y al de Arestis y Demetriades (1999).

Una de las limitaciones del estudio es que ante la carencia de información trimestral de la población, ésta hubo de generarse asumiendo un crecimiento lineal de la misma a lo largo de cada año, lo cual puede estar no muy lejos de la realidad. Quizá otra restricción sea el que se haya empleado una estimación de los acervos de capital agregados en México, ya que no se cuenta con estadísticas oficiales sobre este indicador.

No obstante lo anterior, nuestras estimaciones sugieren una retroalimentación, en términos de causalidad, entre el desarrollo financiero y el crecimiento económico en México, de ahí la necesidad de promover las políticas que tiendan al impulso del desarrollo financiero para aprovechar la relación positiva entre ambas variables, lo cual es sugerido por el resultado de las estimaciones efectuadas en este trabajo.

Encontrar e identificar las políticas apropiadas que contribuyan a la generación del crecimiento económico, así como el estudio de los mecanismos mediante los cuales sea posible lograrlo, destaca la necesidad de reconsiderar la importancia del vínculo entre desarrollo financiero y crecimiento económico.

\section{Conclusiones}

La investigación sobre el desarrollo financiero y crecimiento económico es abundante pero no concluyente respecto de si el sistema financiero es un motor que impulsa el crecimiento económico, si es a la inversa, o bien si se determinan de manera recíproca.

A diferencia de los hallazgos recientes de otros autores, como Tinoco, Torres y Venegas (2008) para el caso de México, nuestros resultados sugieren que el desarrollo financiero incide de manera positiva sobre el crecimiento económico y el análisis de causalidad sugiere una relación bidireccional entre ambas variables, lo cual puede interpretarse como una suerte de círculo virtuoso entre ellas, cuya principal implicación 
es que las autoridades monetarias y financieras deben fomentar y auspiciar políticas que tiendan a desarrollar el sector financiero, para que, a su vez, éste impulse el crecimiento económico de nuestro país.

No obstante, en la función de producción agregada que estimamos para evaluar dicha relación, encontramos que el signo de la relación capital-trabajo es negativo, opuesto al que se esperaba encontrar. Cabe destacar que la negatividad de este signo no es acorde con lo encontrado por Asteriou y Price (2000) en una especificación similar para el caso del Reino Unido.

Desde luego, se requiere una mayor investigación sobre el tema, especialmente en lo que se refiere a los efectos desagregados de los diversos componentes del sector financiero, con la finalidad de averiguar qué actividades contribuyen en mayor proporción al crecimiento económico y, de ser posible, establecer los mecanismos de transmisión más eficaces que están detrás de este importante vínculo.

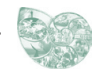

\section{Bibliografía}

Abu-Bader, Suleiman y Aamer Abu-Qarn, "Financial development and economic growth nexus: time series evidence from Middle Eastern and North African countries", Working Paper 223, Ben-Gurion University of the Negev, Department of Economics, 2006.

, "Financial development and economic growth: time series evidence from Egypt", Working Papers 206, Ben-Gurion University of the Negev, Department of Economics, 2005.

Andriesz, Ewa, Dimitrios Asteriou y Keith Pilbeam, "The linkage between financial liberalisation and economic development: Empirical evidence from Poland", City University Economics Discussion Papers 03/03, Londres, Department of Economics School of Social Sciences.

Arestis, Philip y Panicos Demetriades, "Finance and growth: Institutional considerations, financial policies and causality", Zagreb International Review of Economics and Business, vol. 2, núm. 1, Zagreb, Faculty of Economics and Business, University of Zagreb, mayo de 1999, pp. 37-62.

Aspe Armella, Pedro, El camino mexicano de la transformación económica, México, FCE, 1993.

Vol. 40, núm. 159, octubre-diciembre / 2009
Asteriou, Dimitrios y Simon Price, "Financial development and economic growth: Time series evidence for the case of UK", Ekonomia, vol. 4, núm. 2, Cyprus Economic Society and University of Cyprus, invierno 2000, pp. 122-141.

Barro, Robert y Xavier Sala-i-Martín, Economic Growth, Nueva York, McGraw Hill, 1995.

Bhattacharya, P. y M. Sivasubramaniam, "Financial development and economic growth in India: 1970-1971 to 1998-1999", Applied Financial Economics, vol. 13, núm. 12, 2003, pp. 925-929.

Bleaney, Michael y Akira Nishiyama, "Explaining growth: a contest between models", Journal of Economic Growth, vol. 7, núm. 1, 2002, pp. 43-56.

Buffie, Edward, "Financial repression, the New Structuralists, and stabilization policy in semi-industrialized economics", Journal of Development Economics, vol. 14, núm. 3, abril de 1984, pp. 305-322.

Christopoulos, Dimitris y Efthymios Tsionas, "Financial development and economic growth: evidence from panel unit root and cointegration tests", Journal of Development Economics, vol. 73, núm. 1, febrero de 2004, pp. 55-74. 
Cuthbertson, Keith, Mark Taylor y Stephen Hall, Applied Econometric Techniques, Nueva York, Simon and Schuster, 1992.

Dritsakis, Nikolaos y Antonios Adamopoulos, "Financial development and economic growth in Greece: An empirical investigation with Granger causality analysis", International Economic Journal, vol. 18, núm. 4, diciembre de 2004, pp. 547-559.

Enders, Walter, Applied Econometric Time Series, Hoboken, N. J., John Wiley and Sons, 1995.

Fry, Maxwell, "In favour of financial liberalisation", The Economic Journal, vol. 107, núm. 442, Royal Economic Society, mayo de 1997, pp. 754-770.

, "Financial development: Theories and recent experience", Oxford Review of Economic Policy, vol. 5, núm. 4, invierno de 1989, pp. 13-28.

Ghosh, Dilip K., "Foreign exchange dynamics, debt and the "Peso problem", en Dilip K. Ghosh y Edgar Ortiz (eds.), The Changing Environment of International Financial Markets: Issues and Analysis, Nueva York, St. Martin's Press, 1994.

Goldsmith, Raymond, Financial Structure and Development, New Haven, Yale University Press, 1969.

Greenwood, Jeremy y Boyan Jovanovic, "Financial development, growth and the distribution of income", Journal of Political Economy, vol. 98, núm. 5, University of Chicago Press, octubre de 1990, pp. 1076-1107.

Gurley, John y Edward Shaw, "Financial aspects of economic development", American Economic Review, vol. 45, núm. 4, 1995, pp. 515-538.

Güryay, Erdal, Okan Veli Şafakli y Behiye Tüzel, "Financial development and economic growth: Evidence from Northern Cyprus", International Research Journal of Finance and Economics, núm. 8, 2007. Disponible en: http://www.eurojournals.com/5erdal. pdf

INEGI, Sistema de cuentas nacionales de México, Instituto Nacional de Estadística, Geografía e Informática, 2006. Disponible en: http://dgcnesyp.inegi.gob.mx/cgi-win/ bdieintsi.exe/Consultar

, Banco de Información Económica, Instituto Nacional de Estadística, Geografía e Informática, 2005. Disponible en: http:// www.inegi.gob.mx/inegi/default.asp
Kapur, Basant, "Alternative stabilization policies for less-developed economies", Journal of Political Economy, vol. 84, núm. 4, The University of Chicago Press, agosto de 1976, pp. 777-795.

King, Robert y Ross Levine, "Finance and growth: Schumpeter might be right", The Quarterly Journal of Economics, vol. 108, núm. 3, Harvard University's Department of Economics, agosto de 1993a, pp. 717-738.

"Finance, entrepreneurship and growth: Theory and evidence", en Journal of Monetary Economics, vol. 32, núm. 3, diciembre de 1993b, pp. 513-542.

Levine, Ross, “Es importante el sistema financiero?", Crecimiento económico: Teoría, instituciones y experiencia internacional, Bogotá D.C., Banco de La República y Banco Mundial, 1995, pp. 366-384.

Loría, Eduardo y De Jesús, Leobardo, "Los acervos de capital de México. Una estimación, 1980:I-2004:IV”, El trimestre económico, vol. LXXIV (2), núm. 294 abril-junio de 2007, pp. 475-485.

Lucas, Robert, "On the mechanics of economic development", Journal of Monetary Economics, vol. 22, núm. 1, 1988, pp. 3-42.

Lütkepohl, Helmut y Markus Krätzig, Applied Time Series Econometrics, Cambridge, Cambridge University Press, 2004.

Mathesion, Donald, "Financial reform and stabilization policy in a developing economy", Journal of Development Economics, vol. 7, núm. 3, 1980, pp. 359-395.

McKinnon, Ronald, "Financial liberalization in retrospect: interest rate policies in LDC's", en Gustav Ranis y T. Paul Shultz (eds.), The State of Development Economics: Progress and Perspective, Nueva York-Oxford, Basil Blackwell, 1988. , Money and Capital in Economic Development, Washington, DC., The Brookings Institution, 1973.

Mosconi Rocco, Johansen Söeren y Bent Nielsen, "Cointegration analysis in the presence of structural breaks in the deterministic trend", Econometrics Journal, vol. 3, núm. 2, Royal Economic Society, 2000, pp. 216-249.

Murinde, Victor, "Financial markets and endogenous growth: an econometric analysis for Pacific Basin countries", Niels Hermes y Robert Lensink, Financial Development and Economic Growth, Londres, Routledge, 1996, pp. 94-114.

Vol. 40, núm. 159, octubre-diciembre / 2009 
Odedokun, M. O., "Alternative econometric approaches for analyzing the role of the financial sector in economic growth: Timeseries evidence from LDCs", Journal of Development Economics, vol. 50, núm. 1, junio de 1996, pp. 119-135.

Robinson, Joan, "The generalisation of the general theory", en The Rate of Interest and Other Essays, Londres, Macmillan, 1952, pp. 69-142.

Romero de Ávila, Diego, "Finance and growth in the EU: New evidence from the liberalization and harmonization of the banking industry", Working Paper Series 266, Frankfurt, European Central Bank, 2003.

Ros, Jamie, Development Theory and the Economics of Growth, Ann Arbor, The University of Michigan Press, 2000.

Roubini, Nouriel y Xavier Sala-i-Martín, "Financial repression and economic growth", Journal of Development Economics, vol. 39, núm. 1, julio de 1992, pp. 5-30.

Ruiz Porras, Antonio, "Mercados financieros y crecimiento económico en América Latina: un análisis econométrico", Análisis económico, vol. XIX, núm. 40, 2004, pp. 141-165.

Schumpeter, J. A, The Theory of Economic Development, Cambridge, Mass., Harvard University Press, 1911.

Shaw, Edward, Financial Deepening in Economic Development, Nueva York, Oxford University Press, 1973.
Solís, Leopoldo, Crisis económico-financiera 1994-1995, México, El Colegio NacionalFondo de Cultura Económica, 1996.

Stern, Nicholas, "The economics of development: A survey", The Economic Journal, vol. 99, núm. 397, Royal Economic Society, septiembre de 1989, pp. 597-685.

Stiglitz, Joseph E., The Role of the State in Financial Markets, Proceedings of the World Bank Annual Conference on Development Economics, 1993.

Tello, Carlos, Estado y desarrollo económico: México 1920-2006, México, Facultad de Economía-Universidad Nacional Autónoma de México, 2007.

Tinoco, Miguel, Víctor Torres y Francisco Venegas-Martínez, "Deregulation, financial development and economic growth in Mexico: Long-term effects and causality", 1 de julio de 2008. Disponible en SSRN: http://ssrn.com/abstract=1010525

Wijnbergen, Sweder van, "Credit policy, inflation and growth in financially repressed economy", Journal of Development Economics, vol. 13, núms. 1-2, agosto-octubre de 1983, pp. 45-65.

$\mathrm{Xu}$, Zhenhui, "Financial development, investment, and economic growth", en Economic Inquiry, vol. 38, núm. 2, abril de 2000, pp. 331-344. 
DOMINGO RODRÍGUEZ BENAVIDES Y FRANCISCO LÓPEZ HERRERA

APÉNDICE

Cuadro 1

Datos empleados en las estimaciones (valores en logaritmos)

\begin{tabular}{|c|c|c|c|c|c|c|}
\hline Periodo & PIB real & Población & M4 & PIB nominal & K & L \\
\hline Mar-90 & 13.9245 & 18.2410 & 19.1800 & 20.2103 & 14.441 & 16.0019 \\
\hline Jun- 90 & 13.9610 & 18.2460 & 19.2753 & 20.2935 & 14.392 & 16.0249 \\
\hline Sep-90 & 13.9134 & 18.2507 & 19.3501 & 20.3210 & 14.421 & 16.0447 \\
\hline Dic-90 & 13.9923 & 18.2555 & 19.4980 & 20.4616 & 14.453 & 16.0700 \\
\hline Mar-97 & 13.9618 & 18.2602 & 19.5612 & 20.5004 & 14.425 & 16.0800 \\
\hline Jun-97 & 14.0158 & 18.2650 & 19.6387 & 20.5843 & 74.506 & 16.0966 \\
\hline Sep-91 & 13.9466 & 18.2696 & 19.6664 & 20.5492 & 74.486 & 16.1004 \\
\hline Dic-97 & 14.0315 & 18.2743 & 19.7732 & 20.6683 & 74.539 & 16.1230 \\
\hline Mar-92 & 14.0077 & 18.2789 & 19.7815 & 20.6897 & 14.512 & 16.1183 \\
\hline Jun-92 & 14.0386 & 18.2835 & 19.8135 & 20.7514 & 14.535 & 16.1143 \\
\hline Sep-92 & 13.9906 & 18.2880 & 19.8389 & 20.7326 & 74.595 & 16.1144 \\
\hline Dic-92 & 14.0593 & 18.2924 & 19.9432 & 20.8238 & 14.594 & 16.1285 \\
\hline Mar-93 & 14.0376 & 18.2969 & 19.9950 & 20.8384 & 14.581 & 16.1265 \\
\hline Jun-93 & 14.0469 & 18.3014 & 20.0631 & 20.8619 & 14.673 & 16.1271 \\
\hline Sep-93 & 14.0074 & 18.3056 & 20.0979 & 20.8381 & 14.638 & 16.1132 \\
\hline Dic-93 & 14.0810 & 18.3098 & 20.1787 & 20.9287 & 14.772 & 16.1229 \\
\hline Mar-94 & 14.0607 & 18.3140 & 20.2225 & 20.9408 & 14.576 & 16.1255 \\
\hline Jun-94 & 14.1018 & 18.3183 & 20.2251 & 20.9989 & 14.719 & 16.1337 \\
\hline Sep-94 & 14.0525 & 18.3223 & 20.2782 & 20.9612 & 74.688 & 16.1368 \\
\hline Dic-94 & 14.1319 & 18.3263 & 20.4004 & 21.0662 & 74.846 & 16.1468 \\
\hline Mar-95 & 14.0563 & 18.3303 & 20.4117 & 21.1306 & 14.282 & 16.1256 \\
\hline Jun-95 & 14.0053 & 18.3343 & 20.4301 & 21.2115 & 14.437 & 16.1111 \\
\hline Sep-95 & 13.9687 & 18.3381 & 20.4588 & 21.2290 & 14.436 & 16.1039 \\
\hline Dic-95 & 14.0589 & 18.3419 & 20.5831 & 21.3852 & 14.642 & 16.1292 \\
\hline Mar-96 & 14.0569 & 18.3457 & 20.6325 & 21.4610 & 14.358 & 16.1454 \\
\hline Jun-96 & 14.0681 & 18.3495 & 20.6887 & 21.5308 & 14.45 & 16.1621 \\
\hline Sep-96 & 14.0376 & 18.3531 & 20.7455 & 21.5429 & 14.437 & 16.1736 \\
\hline Dic-96 & 14.1276 & 18.3567 & 20.8331 & 21.6790 & 14.638 & 16.2058 \\
\hline Mar-97 & 14.1018 & 18.3603 & 20.8738 & 21.7016 & 14.466 & 16.2258 \\
\hline Jun-97 & 14.1486 & 18.3639 & 20.9394 & 21.7732 & 14.558 & 16.2510 \\
\hline Sep-97 & 14.1097 & 18.3673 & 20.9810 & 21.7614 & 14.588 & 16.3022 \\
\hline Dic-97 & 14.1921 & 18.3708 & 21.0600 & 21.8773 & 14.706 & 16.3421 \\
\hline Mar-98 & 14.1745 & 18.3742 & 21.1083 & 21.9237 & 14.516 & 16.3758 \\
\hline Jun-98 & 14.1909 & 18.3777 & 21.1610 & 21.9635 & 14.661 & 16.3882 \\
\hline Sep-98 & 14.1611 & 18.3811 & 21.2111 & 21.9622 & 14.662 & 16.4027 \\
\hline Dic-98 & 14.2189 & 18.3845 & 21.2974 & 22.0710 & 14.675 & 16.4147 \\
\hline Mar-99 & 14.1958 & 18.3880 & 21.3414 & 22.0999 & 14.522 & 16.4370 \\
\hline Jun-99 & 14.2252 & 18.3914 & 21.3732 & 22.1487 & 14.681 & 16.4493 \\
\hline Sep-99 & 14.2045 & 18.3948 & 21.4229 & 22.1462 & 14.694 & 16.4707 \\
\hline Dic-99 & 14.2712 & 18.3982 & 21.4753 & 22.2449 & 14.723 & 16.4837 \\
\hline Mar-00 & 14.2674 & 18.4016 & 21.5096 & 22.2880 & 14.641 & 16.5033 \\
\hline Jun-00 & 14.2961 & 18.4049 & 21.5451 & 22.3296 & 14.789 & 16.5109 \\
\hline Sep-00 & 14.2726 & 18.4082 & 21.5803 & 22.3746 & 14.807 & 16.5256 \\
\hline Dic-00 & 14.3172 & 18.4114 & 21.6053 & 22.3875 & 14.802 & 16.5294 \\
\hline Mar-01 & 14.2865 & 18.4146 & 21.6397 & 22.3781 & 74.801 & 16.5324 \\
\hline $\begin{array}{ll}\text { Jun-01 } \\
\end{array}$ & 14.2977 & 18.4178 & 21.6631 & 22.3909 & 14.866 & 16.5218 \\
\hline Sep-01 & 14.2595 & 18.4208 & 21.7107 & 22.3523 & 74.881 & 16.5191 \\
\hline Dic-01 & 14.3035 & 18.4238 & 21.7561 & 22.4199 & 14.882 & 16.5212 \\
\hline Mar-02 & 14.2634 & 18.4268 & 21.7685 & 22.4217 & 14.794 & 16.5272 \\
\hline Jun-02 & 14.3166 & 18.4297 & 21.7874 & 22.4821 & 14.901 & 16.5300 \\
\hline Sep-02 & 14.2763 & 18.4324 & 21.7942 & 22.4483 & 14.872 & 16.5387 \\
\hline Dic-02 & 14.3232 & 18.4351 & 21.8537 & 22.5270 & 14.95 & 16.5474 \\
\hline Mar-03 & 14.2867 & 18.4378 & 21.8600 & 22.5291 & 14.783 & 16.5539 \\
\hline Jun-03 & 14.3159 & 18.4405 & 21.8847 & 22.5556 & 14.901 & 16.5506 \\
\hline Sep-03 & 14.2866 & 18.4429 & 21.9125 & 22.5213 & 14.856 & 16.5526 \\
\hline Dic-03 & 14.3441 & 18.4454 & 21.9723 & 22.6141 & 14.948 & 16.5660 \\
\hline Mar-04 & 14.3225 & 18.4478 & 21.9990 & 22.6193 & 14.826 & 16.5809 \\
\hline Jun-04 & 14.3525 & 18.4503 & 22.0108 & 22.6598 & 14.906 & 16.5818 \\
\hline Sep-04 & 14.3303 & 18.4525 & 22.0239 & 22.6402 & 14.845 & 16.5892 \\
\hline Dic-04 & 14.3915 & 18.4548 & 22.0856 & 22.7356 & 14.8980 & 16.6060 \\
\hline
\end{tabular}

\section{Desarroollo}

Vol. 40, núm. 159, octubre-diciembre / 2009 